\title{
Locked Quintessence and Cold Dark Matter
}

\author{
Minos Axenides* \\ *Institute of Nuclear Physics, National Center for Scientific Research 'Demokritos', \\ Agia Paraskevi Attikis, Athens 153 10, Greece
}

\begin{abstract}
A supersymmetric hybrid potential model with low energy supersymmetry breaking scale $\left(M_{S} \sim\right.$ $1-10 T e v$ ) is presented for both dark matter and dark energy. Cold dark matter is associated with a light modulus field $(\sim 10-100 \mathrm{Mev})$ undergoing coherent oscillations around a saddle point false vacuum with the presently observed energy density $\left(\rho_{0} \sim 10^{-12} \mathrm{eV}^{4}\right)$. The latter is generated by its coupling to a light dark energy scalar field $\left(\sim 10^{-18} \mathrm{eV}\right)$ which is trapped at the origin ("locked quintessence"). Through naturally attained initial conditions the model is consistent with cosmic coincidence reproducing LCDM cosmology. An exit from the cosmic acceleration phase is estimated to occur within some eight Hubble times.
\end{abstract}

\section{Introduction}

There is a growing observational evidence to the fact that we live in a spatially flat Universe $\left(\Omega_{t o t} \approx 1\right)$ in a state of cosmic acceleration [1, 2, 3, 4]. Most of its content, by weight $\left(\Omega_{t o t}-\Omega_{b a r} \sim 0.96\right)$, cannot be accounted for by the standard model of particle physics. It is believed to be associated with an invisible sector of Matter and Energy of, remarkably, almost equal energy density in a cosmic coincidence. Dark Matter $\left(\Omega_{D M} \sim 0.3\right)$, responsible for the growth of structure in our Universe, is believed to be non-baryonic in nature with small free streaming length behaving as a non-relativistic gas (Cold Dark Matter-CDM). It is typically associated with weakly interacting massive particles (WIMPs) such as axions, axinos, neutralinos, gravitinos, string moduli and others[5].

Dark Energy $\left(\Omega_{D E} \sim 0.7\right)$, on the other hand, is probably a homogeneous perfect fluid $\operatorname{component}(p \sim$ $w \rho)$ with negative pressure $\left(w<-\frac{1}{3}\right)$ giving rise to the observed cosmic acceleration(for a review see [6]). In its most popular version it is attributed to the Cosmological Constant $(w=-1)$ whose value must be fine tuned to an unprecedent degree to be in accordance with the observational data $\left(\frac{\Lambda}{8 \pi G} \sim 10^{-47}\right)$. The emerging LCDM Cosmology, although economical and succesful is not lacking of theoretical shortcomings. Indeed a constant vacuum energy inevitably leads to eternal accelerated expansion, technically implying the presence of causal horizons and hence non-existence of well defined in and out states in the formulation of the underlying quantum theory such as String theory[7].

Alternative scenarios employ dynamical scalars, such as Quintessence fields $(-1<w<-1 / 3)$ [8] which possess time varying energy density as they roll down their monotonically decreasing potential energies . They typically predict an exit from the present accelerating phase. Eventhough these models dispense with the theoretical problems of the Cosmological Constant scenario they dont lack unnatural fine tunnings[9] associated typically with both their initial conditions, present value and/or their small mass $\left(M_{Q} \sim 10^{-33}\right.$ $\mathrm{eV}$ ). In the context of supergravity theories such a light field is difficult to be understood because the flatness of its potential is lifted by excessive supergravity corrections or due to the action of non-renormalizable terms, which become important at displacements of order $M_{P}$.

Cosmic acceleration in the very early universe has been extensively studied in supersymmetric hybrid models[14]. There the vacuum energy density required in order to generate the necessary number of efoldings is fed into the slow rolling inflaton through its coupling to a second scalar field the "waterfall" which is kept trapped along the inflaton track. In a fast-roll variation of this scenario, also dubbed "locked inflation" [11, 12], the inflaton field undergoes rapid coherent oscillations around its "Saddle point" vacuum before it is displaced away from it, prolonging consequently the inflationary phase.

Interestingly it has been known for quite a while that coherent oscillations of massive (pseudo)scalar weakly interacting particles, such as the axion can mimic Cold Dark Matter $(w=0)$ [15]. We have recently produced an interacting model that realizes LCDM Cosmology by putting these two ingredients together in the very late universe [13]. Other interacting models for Dark Matter and Dark Energy can be found in Ref. [10]. 
Our model is a given by a standard Supersymmetric Hybrid Potential with only two characteristic energy scales : the Planck Mass $\left(M_{P l} \approx 10^{19} \mathrm{GeV}\right)$ and a low energy SUSY breaking scale $\left(M_{S} \approx M_{3 / 2} \approx 1 T e V\right)$. We assume that the dark matter particle is a modulus $\Phi$, corresponding to a flat direction of supersymmetry. The modulus field is undergoing coherent oscillations, which are equivalent to a collection of massive $\Phi-$ particles $\left(M_{\Phi}=\frac{M_{S}^{2}}{M_{P l}} \sim 10-100 \mathrm{MeV}\right)$, that are the required WIMPs. A second scalar field $(\Psi)$ interacts with $(\Phi)$ in a standard way $\left(\lambda \Phi^{2} \Psi^{2}\right)$. This can be thought of as our quintessence field and it corresponds to a flat direction lifted by non-renormalizable terms. Even though the $\Psi$-field is a light scalar $\left(M_{\Psi} \approx \frac{M_{S}^{3}}{M_{P l}^{2}} \approx 10^{-18}\right.$ $\mathrm{eV}$ ), it is much more massive than the $m_{Q}$ mentioned above, so as not to be in danger from supergravity corrections to its potential[16, 17]. Our quintessence field is coupled to our dark matter in a hybrid manner, which is quite natural in the context of a supersymmetric theory. Due to this coupling, the oscillating $\Phi$, keeps $\Psi$ 'locked' on top of a potential hill, giving rise to the desired dark energy. When the amplitude of the $\Phi$-oscillations decreases enough, the dark energy dominates the Universe, causing the observed accelerated expansion as dictated by the cosmic coincidence. Within some eight Hubble times, when the oscillating amplitude falls below the width of the saddle, the 'locked' quintessence field is released and rolls down to its global minimum. The system reaches the true vacuum and accelerated expansion ceases.

We assume a spatially flat Universe, according to the WMAP observations [1]. We use natural units such that $\hbar=c=1$ and Newton's gravitational constant is $8 \pi G=M_{P l}^{-2}$, where $M_{P l}=10^{18} \mathrm{GeV}$ is the reduced Planck mass.

\section{A Supersymmetric Hybrid Model}

Consider two real scalar fields $\Phi$ and $\Psi$ interacting through a hybrid type of potential of the form[13]

$$
V(\Phi, \Psi)=\frac{1}{2} m_{\Phi}^{2} \Phi^{2}+\frac{1}{2} \lambda \Phi^{2} \Psi^{2}+\frac{1}{4} \alpha\left(\Psi^{2}-M_{s}^{2}\right)^{2},
$$

where $(\lambda \leq 1)$ and $\left(\alpha=\frac{M_{s}^{4}}{M_{P l}^{4}}\right)$. Dark Matter is associated with $\Phi$ and Dark Energy with $\Psi$. All parameters are expressed in terms of two fundamental energy scales: the Planck mass $\left(M_{P l} \sim 10^{18} \mathrm{GeV}\right)$ and the Susy breaking scale which is also taken to be the Gravitino mass $\left(M_{s} \sim m_{3 / 2} \sim 1 \mathrm{TeV}\right)$. They should be considered in the framework of gauge mediated supersymmetry[18]. Standard features of the potential which is depicted in the figure are:

- Two global minima $(\Phi, \Psi)=\left(0, M_{s}\right)$ with an unstable saddle point at $(\Phi, \Psi)=(0,0)$

- $\Psi$ possesses a $\Phi$ dependent curvature $\left.\left(m_{\Psi}^{\text {eff }}\right)^{2}=\lambda \Phi^{2}-\alpha M_{s}^{2}\right)$ with a width of

$$
\Phi_{w}=\frac{1}{\sqrt{\lambda}} \frac{M_{s}^{3}}{M_{P l}^{2}}
$$

- Cosmic Coincidence $\left(\Omega_{D E}-\Omega_{D M} \sim \mathcal{O}(1)\right)$ at present Hubble time demands small scalar masses : $m_{\Phi} \sim \frac{M_{s}^{2}}{M_{P l}} \approx 10-100 \mathrm{MeV}$ and $m_{\psi} \sim \frac{M_{s}^{3}}{M_{P l}^{2}} \approx 10^{-18} \mathrm{eV}$. The latter is conceivable to be due to accidental cancellations in the Kähler potential or some other accidental symmetry protecting $m_{\Psi}$.

Our physical system acts as a two component perfect fluid with energy density $\left(\rho_{t o t}=\rho_{\Phi}+\rho_{\Psi}\right)$ which gets diluted as the universe expands. When the system finds itself rolling at $\Phi \geq \Phi w$ it is energetically favorable for $\Psi$ to be trapped in its origin $\Psi \cong 0$. $\Phi$ performs coherent oscillations in a quadratic potential

$$
V(\Phi, \Psi=0)=\frac{1}{2} m_{\Phi}^{2} \Phi^{2}+V_{0}
$$

around a saddle point false vaccuum with energy density given by

$$
V_{0}=\frac{1}{4} \alpha M_{s}^{4} \sim \frac{M_{s}^{8}}{M_{P l}^{4}} \sim 10^{-120} M_{P l}^{4}
$$




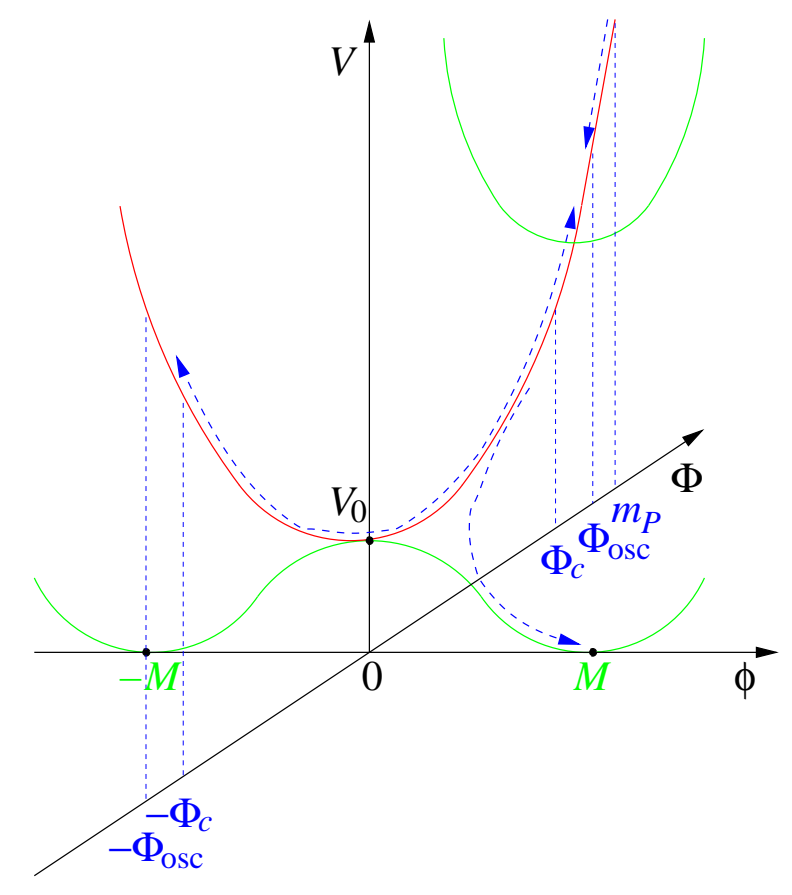

Figure 1: Illustration of the scalar potential $V(\Phi, \Psi)$. Originally, $\Phi \sim M_{P l}$ and $\Psi \simeq 0$. The field $\Phi$ begins oscillating with amplitude $\Phi_{\mathrm{osc}}$. Due to the expanding Universe its energy gets diluted until it reaches $\bar{\Phi}_{\text {end }} \sim \Phi_{w}$, when the system departs from the saddle and rolls toward the minimum at $\left(\Phi=0, \Psi= \pm M_{s}\right)$.

the observed present vacuum energy density. It is associated with the DE condensate $\Psi$ acting as an effective cosmological constant, i.e. behaving as a perfect fluid component with an equation of state $\left(p=-V_{0}\right)$. In the high temperature phase the energy density is dominated by the kinetic energy of the $\Phi$ oscillations which behaves as a pressureles non-relativistic component of a collection of massive particles, hence Cold Dark Matter(CDM), which is given by :

$$
\rho_{\Phi}=\frac{1}{2} \dot{\Phi}^{2}+\frac{1}{2} m_{\Phi}^{2} \Phi^{2}
$$

where the dot denotes derivative with respect to the cosmic time $t$. The model therefore identifies the following cosmic phases:

CDM domination The overall density is dominated by the coherent oscillations of $\Phi$ in Eq. (5), when the oscillation amplitude is larger than

$$
\Phi_{\Lambda} \sim \frac{\sqrt{\alpha} M^{2}}{m_{\Phi}} \sim\left(\frac{m_{\Psi}}{m_{\Phi}}\right) M_{s} \sim \frac{M_{s}^{2}}{M_{P l}}
$$

They behave as a collection of non-relativistic particles whose energy gets diluted accordingly as $\left(\rho_{\Phi} \propto\right.$ $\left.R^{-3}\right)$

Locked Quintessence The energy density is dominated by the Saddle Point Vacuum of eq.(4) for the range of $\Phi$ amplitude oscillations

$$
\Phi_{w}<\Phi_{0}<\Phi_{\Lambda}
$$

The characteristic time scale that $\Phi$ spends on the saddle $\left(\Phi_{0}<\Phi_{w}\right)$ is $\left(\Delta t_{w} \sim \frac{\Phi_{w}}{m_{\Phi} \Phi_{0}}\right)$. As long as it is smaller than the time scale $\left(\Delta t_{\Psi} \approx \frac{1}{m_{\Psi}}\right)$ it takes for $\Psi$ to start to roll away from the top of the hill rapid coherent oscillations of $\Phi$ persist (Locked Quintessence). The effect is present due to the ratio of masses chosen $\left(\frac{m_{\Phi}}{m_{\Psi}} \approx \frac{M_{P l}}{M_{s}} \approx 10^{16}\right)$. A (quasi) de Sitter expansion phase sets in with $a \simeq a_{0} \exp \left(H_{0} \Delta t\right)$, where $\Delta t=t-t_{0}$ and $H_{0} \simeq \sqrt{V_{0}} / \sqrt{3} M_{P l}=$ constant. For the oscillating $\Phi$ we have $\Phi \propto \sqrt{\rho_{\Phi}} \propto a^{-3 / 2}$. We can thus obtain an estimate of the length of the cosmic acceleration phase. 
Post-Acceleration Phase Our two fluid system will release its stored vacuum energy when $\Psi$ will start to roll away from the top of the hill away from its present false vacuum state into its future true vacuum of zero energy density when $\left(\Phi_{0}=\Phi_{w}=\Phi_{\Lambda}\right)$

$$
\Phi_{w} \simeq \Phi_{\Lambda} \exp \left(-\frac{3}{2} H_{0} \Delta t_{w}\right) \Rightarrow \Delta t_{w} \simeq \frac{2}{3}\left[\ln \left(\frac{M_{P l}}{M_{S}}\right)+\ln \sqrt{\lambda}\right] H_{0}^{-1},
$$

We see that the period of acceleration may last up to wight Hubble times (e-foldings) depending on the value of $\lambda$.

\section{$3 \quad$ Dark Matter and Dark Energy Requiremenets}

- Coherent Oscillations of the modulus $\Phi$ field in a quadratic potential behave as a collection of massive non-relativistic particles. In order that we may identify them with a realistic CDM component $\left(\Omega \approx \frac{1}{3}\right)$ they must persist until today with the $\Phi$ quanta not having decayed, namely satisfying

$$
\Gamma_{\Phi}<H_{0}
$$

where $H_{0} \sim \sqrt{\rho_{0}} / M_{P l}$ is the Hubble parameter at present. Using that $\Gamma_{\Phi} \sim g_{\Phi}^{2} m_{\Phi}$ we find the bound

$$
m_{\Phi} \leq 10^{-20} M_{P l}
$$

where we used that the coupling $g_{\Phi}$ of $\Phi$ with its decay products lies in the range $\frac{m_{\Phi}}{M_{P l}} \leq g_{\Phi} \leq 1$, with the lower bound corresponding to the gravitational decay of $\Phi$, for which $\Gamma_{\Phi} \sim\left[m_{\Phi}^{3}\right] / M_{P l}^{2}$. We may conclude that $\Phi$ has to be a rather light field with mass $\lesssim 10-100 \mathrm{MeV}$.

- We must require that our dark matter field $\Phi$ should not decay into $\Psi$-particles, through their mutual coupling, until the present time either perturbatively $(\Phi \rightarrow \phi \phi)$ or non-perturbatively through parametric resonance. The perturbative condition reads

$$
\Gamma_{\Phi \rightarrow \phi \phi} \simeq \frac{\lambda^{2} \Phi_{0}^{2}}{8 \pi m_{\Phi}}<H_{0} .
$$

Since $\bar{\Phi} \propto a^{-3 / 2}$, it becomes obvious that the above constraint is the tightest in the early times after the amplitude of oscillations become $\left(\Phi_{0} \approx \frac{m_{\Phi}}{\sqrt{\lambda}}\right)$ which takes place in the radiation era. By imposing it we get an upperbound condition for $\lambda$ :

$$
\lambda<\frac{m_{\Phi}}{M_{P l}}\left(\frac{M_{P l}}{T_{\mathrm{eq}}}\right)^{2 / 5} \sim 10^{-19} .
$$

- The condition that the oscillations of $\Phi$ are dominated by $V_{0}$ of eq. 4 in the present Hubble era imply that $\left(\Phi_{0} \leq \Phi_{\Lambda}\right)$ gets to be satisfied when $\left(\sqrt{\lambda}\left(\frac{m_{P l}}{M_{s}}\right)>1\right)$ which, in turn, gives us a lower bound for the coupling constant

$$
\lambda>10^{-30} .
$$

- The onset of $\Phi$-oscillations must occur in the radiation era $(T>1 \mathrm{eV})$ when $\left(H_{o s c} \sim m_{\Phi}\right)$ in the aftermath of an early phase of inflation being followed right afterwards by reheating[19]. Their fractional contribution to the energy density is $\left(\frac{\rho_{\Phi}}{\rho} \propto \alpha \propto H^{-\frac{1}{2}}\right)$. They eventually dominate the energy density of the Universe. By requiring this to take place at $\left(T_{e q}=1 \mathrm{eV}\right)$ we find the initial displacement of $\Phi$ to be much smaller than the Planck scale namely $\left(\Phi_{o s c} \sim 10^{-6} M_{P l} \ll M_{P l}\right)$. However the inclusion of supergravity corrections to the potential $\left(\Delta m_{\Phi}^{2} \propto H(t)^{2}\right)[20]$ lift the flatness of the $\Phi$ direction so that $\Phi$ begins to roll down long before $\left(H \sim m_{\Phi}\right)$. Its motion is, however, overdamped by the excessive friction of a large Hubble parameter (compared to its mass) imposing a freeze out to the value of $\Phi$ until $\mathrm{H}$ is reduced enough for the quadratic oscillations to commence. 
- Similar in spirit analysis can be applied to the study of the initial conditions for the Quintessence field $\Psi$ which has to find itself near the origin $\left(\Psi \leq M_{s}\right)$ in order to get "locked" when the $\Phi$ oscillations begin. The oscillations of $\Psi$ begin immediately after reheating with $\left(\Psi \propto \sqrt{\rho_{\Psi}} \propto H^{3 / 4}\right)$. It can be analytically demonstrated that our original assumption for $(\Psi \approx 0)$ is well justified.

- The smallness of the saddle point vacuum energy does not only require a small mass for our tachyonic field $\Psi$ but a small VEV as well $\left(M_{s} \sim 1 \mathrm{TeV}\right)$. This can be done through higher order nonrenormalizable terms or logarithmic loop corrections[16]. Clearly the level of fine tuning implied by $\left(m_{\Phi} \sim 10^{15} H_{0} \sim 10^{9} H_{e q}\right)$ is much less severe than the one required in most quintessence models $\left(m_{Q} \sim H_{0}\right)$. As a consequence and in contrast to quintessence models Sugra corrections in the matter era are negligible.

\section{Conclusions}

We have presented a unified model of dark matter and dark energy in the context of low-scale gauge-mediated supersymmetry breaking. Our LQCDM model retains the predictions of LCDM Cosmology, while avoiding eternal acceleration and achieving coincidence without significant fine-tuning. The initial conditions of our model are naturally attained due to the effect of supergravity corrections to the scalar potential in the early Universe, following a period of primordial inflation. Our oscillating $\Phi$-condensate does not have to be the dark matter necessarily. Indeed, it is quite possible that $\Psi$-remains locked on top of the false vacuum while $\rho_{\Phi}$ is negligible at present. It is easy to see that indeed $\left(\frac{\rho_{\Phi}^{\min }}{\rho_{0}} \sim 10^{-30} \lambda^{-1}\right)$. Depending on $\lambda$, $\Phi$ may contribute only by a small fraction to dark matter at present, while still being able to lock quintessence and cause the observed accelerated expansion at present. This option appears less appealing to us.

\section{References}

[1] D. N. Spergel et al., Astrophys. J. Suppl. 148, 175 (2003).

[2] M. Tegmark et al. [SDSS Collaboration], Phys. Rev. D 69 (2004) 103501

[3] M. Colless, astro-ph/0305051.

[4] S. Perlmutter et al. [Supernova Cosmology Project Collaboration], Astrophys. J. 517, 565 (1999); A. G. Riess et al. [Supernova Search Team Collaboration], Astron. J. 116, 1009 (1998).

[5] G. Jungman, M. Kamionkowski and K. Griest, Phys. Rept. 267 (1996) 195 J. R. Primack, D. Seckel and B. Sadoulet, Ann. Rev. Nucl. Part. Sci. 38 (1988) 751.

[6] P. J. E. Peebles and B. Ratra, Rev. Mod. Phys. 75, 559 (2003).

[7] S. Hellerman, N. Kaloper and L. Susskind, JHEP 0106, 003 (2001); W. Fischler, A. Kashani-Poor, R. McNees and S. Paban, JHEP 0107, 003 (2001); E. Witten, hep-th/0106109; N. Goheer, M. Kleban and L. Susskind, JHEP 0307, 056 (2003).

[8] L. M. Wang, R. R. Caldwell, J. P. Ostriker and P. J. Steinhardt, Astrophys. J. 530, 17 (2000); I. Zlatev, L. M. Wang and P. J. Steinhardt, Phys. Rev. Lett. 82, 896 (1999); G. Huey, L. M. Wang, R. Dave, R. R. Caldwell and P. J. Steinhardt, Phys. Rev. D 59, 063005 (1999); R. R. Caldwell, R. Dave and P. J. Steinhardt, Phys. Rev. Lett. 80, 1582 (1998).

[9] C. F. Kolda and D. H. Lyth, Phys. Lett. B 458, 197 (1999).

[10] R. Bean and J. Magueijo, Phys. Lett. B 517, 177 (2001); L. Amendola and D. Tocchini-Valentini, Phys. Rev. D 64 (2001) 043509; D. Tocchini-Valentini and L. Amendola, Phys. Rev. D 65, 063508 (2002); W. Zimdahl and D. Pavon, Phys. Lett. B 521, 133 (2001); M. Pietroni, Phys. Rev. D 67, 103523 (2003); T. Padmanabhan and T. R. Choudhury, Phys. Rev. D 66, 081301 (2002); D. Comelli, M. Pietroni and A. Riotto, Phys. Lett. B 571, 115 (2003); H. Ziaeepour, Phys. Rev. D 69 (2004) 063512; G. R. Farrar and P. J. E. Peebles, astro-ph/0307316; E. I. Guendelman and A. B. Kaganovich, gr-qc/0312006. R. G. Cai and A. Wang, arXiv:hep-th/0411025. 
[11] G. Dvali and S. Kachru, hep-th/0309095; hep-ph/0310244. R. Easther, J. Khoury and K. Schalm, JCAP 0406 (2004) 006 [arXiv:hep-th/0402218].

[12] K. Dimopoulos and M. Axenides, arXiv:hep-ph/0310194.

[13] M. Axenides and K. Dimopoulos, JCAP 0407 (2004) 010

[14] A. D. Linde, Phys. Rev. D 49 (1994) 748 L. Randall, M. Soljacic and A. H. Guth, Nucl. Phys. B 472 (1996) 377 G. Lazarides, arXiv:hep-ph/0011130. M. Berkooz, M. Dine and T. Volansky, arXiv:hep$\mathrm{ph} / 0409226$.

[15] M. S. Turner, Phys. Rev. D 28, 1243 (1983) S. D. H. Hsu, Phys. Lett. B 567 (2003) 9

[16] A. R. Liddle and D. H. Lyth, Cosmological Inflation and Large-Scale Structure (Cambridge Univ. Press, Cambridge U.K., 2000).

[17] M. Dine, L. Randall and S. Thomas, Nucl. Phys. B 458, 291 (1996); Phys. Rev. Lett. 75, 398 (1995).

[18] G. F. Giudice and R. Rattazzi, Phys. Rept. 322 (1999) 419

[19] L. Kofman, A. D. Linde and A. A. Starobinsky, Phys. Rev. D 56 (1997) 3258.

[20] D. H. Lyth and T. Moroi, JHEP 0405 (2004) 004. 